\title{
Demyelinating Polyneuropathy
}

National Cancer Institute

\section{Source}

National Cancer Institute. Demyelinating Polyneuropathy. NCI Thesaurus. Code C27062.

Polyneuropathy that is characterized by demyelination of axons. 